\title{
S-Transform Based Novel Indices for Power Quality Disturbances
}

\author{
Zhengyou He and Yong Jia \\ Southwest Jiaotong University
}

China

\section{Introduction}

Power quality (PQ) has recently become an increasing concern for electric utilities and their customers due to the ever-growing proliferation of power electronic devices and nonlinear loads in electrical power networks. The opening of power markets, and the deregulation and restructuring throughout the world are further changing the framework in which power quality is addressed (Beaulieu et al., 2002). Therefore, the techniques for power quality study and power disturbance mitigation are capturing increasing attention, and consequently, manufacturers are integrating power quality monitoring functions in their products such as power meters, digital relays and event recorders (Ward, 2001). One of the most important aspects in power quality analysis is to evaluate the extent of power disturbances and their negative impacts on power systems. Power quality indices, as a powerful tool for quantifying power quality disturbances, are the concise numerical representations characterizing the nature based on the time and frequency information of the disturbance waveform (Lin \& Domijan, 2005). They also serve as the basis for illustrating the negative impacts of electrical disturbances and for assessing compliance with the required standards or recommendations within a given regulatory framework.

A power quality disturbance usually involves a variation in the electric voltage or current and can be classified based on the waveform time-statistical characteristics into two groups: steady disturbances (harmonic distortion, unbalance, flicker etc.) and transient disturbances (voltage sags, voltage swells, impulses, oscillatory transient etc.). Several power quality indices identified and utilized in the past were accepted and worked well for single and three-phase balanced systems and with periodic stationary waveforms, however, pitfalls of these indices in common use are discussed in (Heydt, 1998, 2000). With the severe changes in the waveforms of the current and voltage signals in the power network, the traditional definitions of the previous indices are no longer valid. As transient disturbances are characterized by spectral components that are significantly time varying in amplitude and/or in frequency; it is important to accommodate the time information in the indices calculation so that they can provide more sensitive interpretation of the disturbances.

Recently, one of the most interesting applications is the assessment of power quality by redefining new indices for transient disturbances using signal processing techniques. New power quality indices have been suggested based on the short time Fourier transform (STFT) (Jaramillo et al., 2000), time frequency distribution(TFD)(Shin et al., 2006) and stationary wavelet transform(SWT)(Morsi \& Hawary, 2008). The integrating of the time 
information into the calculation of several power quality indices using STFT is addressed in (Jaramillo et al., 2000); however, although the STFT technique offered a partial solution for the time information, it has limitations due to its fixed window length, which has to be chosen prior to the analysis. This drawback is reflected in the achievable frequency resolution when analyzing non-stationary signals with both low and high-frequency components (Gargoom et al., 2005). Wavelet transform (WT) presents a signal in the timefrequency plane using adaptive windows and Mallat fast algorithm. However, WT is not suitable for analyzing low frequency disturbance, in addition, some importance frequency components of the disturbance are not extracted precisely due to the non-adjustable central frequency and frequency bands once the sampling frequency is determined (Lin \& Domijan, 2005). The various types of time frequency distributions namely "Cohen' class" synthetically, are a bilinear transformation providing simultaneous time and frequency information on the energy content of the signal. As the disturbances in power systems are characterized by the presence of multiple frequency components over a short duration, interference is also problematic.

Recently, to overcome the limitations of the previous techniques, the previous works (Stockwell et al., 1996, Mishra et a., 2008, Zhan et al. 2005, Chilukuri \& Dash, 2004) introduce the S-transform (ST) as a new power quality analysis technique. This chapter is concerned with investigating the power quality indices obtained in the S-transform domain for transient disturbances assessment. The chapter is organized as follows. Section X.2 discusses the theoretical background of STFT, WT and S-transform. The indices that are most frequently used in international standards and recommendations are introduced in section X.3. Section X.3 will also define four new power quality indices for transient disturbances based on S-transform. Then, section IV discusses the performance of these indices on simulated power quality signals. Finally, conclusions are given in Section V.

\section{Theoretical background}

Signal processing techniques have been widely used for automatic power quality (PQ) disturbance detection and recognition (Bollen \& $\mathrm{Gu}, 2006$ ). As for power quality assessment, these high-performance signal-analysis techniques are potential to calculate PQ indices based on the knowledge of what spectral components are present in the signal and where they are located in time (time frequency representations). In this section, the background theories of the STFT, WT and S-transform are presented.

\subsection{Short-time Fourier Transform}

As is well-known, the Fourier Transform is the main tool for signal spectral decomposition. The Fourier transform (FT) of a s signal $x(t)$ with $t \in(-\infty,+\infty)$ is defined by Equation(1) below:

$$
\mathrm{FT}(f)=\int_{-\infty}^{+\infty} x(t) \mathrm{e}^{j 2 \pi f t} d t
$$

In the discrete time domain, the Fourier transform of a discrete sampled waveform $x(n)$ is defined as follows:

$$
\mathrm{FT}(f)=\sum_{n=-\infty}^{\infty} x(n) \mathrm{e}^{j 2 \pi f n T}
$$


In practical applications, time domain waveforms are finite sequences of samples. If a finite sequence of L samples of the waveform $x(n), n=0,1, \ldots, \mathrm{L}-1$, is considered, its L-point DFT is given by:

$$
\operatorname{DFT}(k)=\sum_{n=0}^{L-1} x(n) \mathrm{e}^{j 2 \pi k n / L} \quad k=0,1, \ldots, L-1
$$

To improve the analysis of signals whose spectrum changes with time we introduce the widely used and well-defined short-time Fourier transform. The STFT is a simple extension of the FT of a general signal $x(t)$, where the FT is evaluated repeatedly for a windowed version of the time domain signal. In practice, each FT gives a frequency domain 'slice' associated with the time value at the centre of the window. With such an approach, the waveform can be represented in a three-dimensional plot so it is possible to know what spectral components are present in the signal and where they are located in time (time frequency representation). In the continuous time domain, STFT is defined by:

$$
\operatorname{STFT}(f, \tau)=\int_{-\infty}^{+\infty} x(t) w(t-\tau) \mathrm{e}^{j 2 \pi f t} d t
$$

where $\mathrm{w}$ is the sliding time window of length $\mathrm{T}_{\mathrm{w}}$, and $\tau$ is the time to which the spectrum refers. In the discrete time domain, Equation (4) becomes:

$$
\operatorname{STDFT}(k)=\sum_{n=0}^{L-1} x(n) w(n-m) \mathrm{e}^{j 2 \pi k n / L} \quad k=0,1, \ldots, L-1
$$

where $x(n)$ and $\mathrm{w}(n)$ are the sampled versions of the signal and of the sliding time window, respectively, $\mathrm{L}$ is the number of samples and $\mathrm{m}$ is related to the discrete time to which the spectrum refers.

The shape of the sliding time window can influence the spectral component values. The choice of the window (e.g. rectangular, Gaussian, Hanning) is therefore an important issue, and this topic has been discussed extensively in the literature when power quality disturbances are dealt with (Gallo, et al., 2002, 2004). In practice, the rectangular window is used frequently when the issue of power quality indices is considered. The STFT has been used as a basis for the development of a short term harmonic distortion (STHD) index in (Jaramillo et al., 2000). Similar extensions for other waveform distortion indices, such as the $\mathrm{K}$-factor and the crest factor, have been considered.

\subsection{Wavelet transform}

The wavelet transform (WT) has been proposed more and more for processing power quality disturbances. It, like the STFT, is a form of time frequency technique since it allows the simultaneous analysis of waveforms in both time and frequency domains. The main advantage of WT over the STFT is the possibility of conducting multi-resolution time frequency analyses, which allows different resolutions of an analyzed spectrum to be obtained at different frequencies.

The continuous wavelet transform (CWT) of a given signal $x(t)$ with respect to the mother wavelet $\psi_{a, b}(t)$ is the following dot product:

$$
\operatorname{CWT}(a, b)=\left\langle x, \psi_{a, b}\right\rangle=\int_{-\infty}^{+\infty} x(t) \frac{1}{\sqrt{a}} \psi^{*}\left(\frac{t-b}{a}\right) d t
$$




$$
\psi_{a, b}(t)=\frac{1}{\sqrt{a}} \psi\left(\frac{t-b}{a}\right)
$$

where * means the complex conjugate. $\psi_{a, b}(t)$ is called the mother wavelet with $a \in \mathrm{R}+$ and $b$ $\in \mathrm{R}$. The wavelet $\psi_{a, b}(t)$ can be dilated (stretched) and translated (shifted in time) by adjusting the two parameters that characterize it.

For an assigned pair of parameters $a$ and $b$, the coefficient obtained by Equation (6) represents how well the signal $x(t)$ and the scaled and translated mother wavelet match. By varying the values of $\mathrm{a}$ and $\mathrm{b}$, we can obtain the wavelet representation of the waveform $x(t)$ in time (corresponding to the $b$ coefficient) and frequency (corresponding to the $a$ coefficient) domains with respect to the chosen mother wavelet.

In order to obtain discrete scale and time parameters, the wavelet function can choose discrete scaling and translation parameters such as $a=a_{0}^{j}$ and $\mathrm{b}=k b_{0} a_{0}^{j}$ where $k$ and $j$ are integers and $a_{0}>1, b_{0}>0$ are fixed. The result is:

$$
\psi_{j, k}(t)=\frac{1}{\sqrt{a_{0}^{j}}} \psi\left(\frac{t-k b_{0} a_{0}^{j}}{a_{0}^{j}}\right)
$$

The wavelet transform of a continuous signal $x(t)$ using discrete scale and time parameters of wavelets leads to the following wavelet coefficients:

$$
\operatorname{CWT}(j, k)=\left\langle x, \psi_{j, k}\right\rangle=\frac{1}{\sqrt{a_{0}^{j}}} \int_{-\infty}^{+\infty} x(t) \psi^{*}\left(\frac{t-k b_{0} a_{0}^{j}}{a_{0}^{j}}\right) d t
$$

The most delicate steps in WT analysis are the choice of the mother wavelet and the choice of the number of expansion levels. With reference to the choice of the mother wavelet, only knowledge of the phenomena to be analyzed and experience can help to make the best choice and often several trials are necessary to obtain the best choice. Just as when making the choice of an appropriate mother wavelet, knowledge of the phenomena to be analyzed and experience are necessary in order to make the best choice of the number of decomposition levels.

\subsection{S-transform}

S-transform is an extension of the ideas of the continuous wavelet transform (CWT) based on a moving and scalable localizing Gaussian window, so it can be considered as generation of the combination of CWT and STFT. The S-Transform $S(\tau, f)$ of a function is $x(t)$ defined as follows.

$$
\begin{gathered}
S(\tau, f)=\int_{-\infty}^{\infty} x(t) w_{f}(\tau-t) e^{-i 2 \pi f t} d t \\
w_{f}(\tau-t)=\frac{|f|}{\sqrt{2 \pi}} e^{-\frac{f^{2}(\tau-t)^{2}}{2}}
\end{gathered}
$$

Where, $w_{f}(\tau-t)$ is the Gaussian window function. $\tau$ is the shift parameter which can adjust the position of the Gaussian window in the time axis. $f$ is the scale parameter. 
Define $X(f)$ as the Fourier transient result of $x(t)$.Then the relationship between S-transform and Fourier transient can be described as follows.

$$
X(f)=\int_{-\infty}^{\infty} S(\tau, f) d \tau
$$

The equation above shows that the time average of the local spectral representation should result identically in the complex-valued global Fourier Spectrum. S-transform can also be written as function of $X(f)$ as

$$
S(\tau, f)=\int_{-\infty}^{\infty} X(v+f) e^{-2 \pi^{2} v^{2} / f^{2}} e^{j \pi v \tau} d v
$$

The discrete version of the S-transform is calculated by taking the advantage of the efficiency of the fast Fourier transform (FFT) and the convolution theorem. The discrete STransform of $x[\mathrm{kT}]$ is obtained by making f equal to $\mathrm{n} / \mathrm{NT}$ and $\tau$ equal to $k \mathrm{~T}$ :

$$
S\left[k T, \frac{n}{N T}\right]=\sum_{m=0}^{N-1} X\left[\frac{m+n}{N T}\right] e^{-\frac{2 \pi^{2} m^{2}}{n^{2}}} e^{j \frac{2 \pi n}{N} k}
$$

where $\mathrm{N}$ is the length of the analyzed signal, the index $k, \mathrm{~m}$ and $\mathrm{n}$ are equal to $0,1 \ldots \mathrm{N}-1$. $\mathrm{T}$ is the time interval between two consecutive samples. The above equation represents a two dimensional matrix called the S-matrix in which the row is corresponding with frequency and the column is corresponding with time. Each column, thus, represents the local spectrum at one point of time and each element of the S-matrix is complex valued corresponding with the amplitude.

The S-transform produces a time-frequency representation of a time series. It uniquely combines a frequency-dependent resolution that simultaneously localizes the real and imaginary spectra. Compared with STFT, the advantage of S-transform is that the width and the height of the time-frequency window vary with the frequency. Like wavelet transform, Stransform is suitable for analyzing non-stationary signals because of its excellent performance of local time-frequency analysis, which means that it has higher frequency resolution in low frequency bands and lower time resolution in high frequency bands. Because of the combination of absolutely referenced phase information and frequency invariant amplitude of the S-transform, one can directly extract the fundamental and harmonic components of the signal from the S-matrix. In the next section, several novel power quality indices will be introduced based on the good time-frequency localization properties of S-transform.

\section{Power quality indices}

Power quality indices are a few representative numbers that are the result of characterizing, reducing, or extracting from a large volume of power quality measurement data. As much as feasible, the number of quality indices should be kept at their minimum, they should also be easy to assess and be representative of the actual impact of the disturbances they characterize. From the above considerations, it can see that there is a need for common power quality indices in order, for different system operators, to measure and report power quality disturbances quality in a consistent and harmonized manner, either to their clients or to the regulators. In this section, an overview of several traditional indices is provided firstly and shortcomings of these indices are discussed, then four new indices are defined for transient disturbance assessment. 


\subsection{Traditional power quality indices}

The most frequently encountered power disturbances, which are low frequency and high frequency phenomena, are classified into several categories in (IEEE Std. 1159). The existing indices for each disturbance are as follows.

\section{A. Waveform distortions}

Waveform distortions (voltage or current) are mainly characterized by the total harmonic distortion factor (THD), which is defined as the RMS of the harmonic content divided by the RMS value of the fundamental component, usually multiplied by 100 . Harmonic index is based on the spectrum of voltage or current over a given window of time. Various methods for obtaining the spectrum are being discussed in the technical literature, but the method almost exclusively used in power quality monitoring is the Fourier transform. A number of international standard documents define the measurement process, including (EN 50160, IEC 61000-3-6, IEC 61000-4-7, IEC 61000-4-30).

\section{B. Slow voltage variations}

Slow voltage variations are usually quantified by calculation of the RMS value of the supply voltage. In assessing RMS supply voltage, measurement has to take place over a relatively long period of time to avoid the instantaneous effect on the measurement caused by individual load switching (e.g. motor starting, inrush current) and faults. (IEC 61000-4-30) defines the procedure for the RMS voltage measurements and, as in (EN 50160, 2000), assigns the 10-min RMS value of the supply voltage (short interval value) to quantify slow voltage variations and considers a week as the minimum measurement period.

\section{Unbalances}

The severity of voltage unbalances is often quantified by means of the voltage unbalance factor, which is defined as the ratio between the negative-sequence voltage component and the positive-sequence voltage component, usually expressed as a percentage. All the standards and guidelines usually consider a week to be the minimum measurement period. The whole measurement and evaluation procedure for the short and long interval values of the negative-sequence voltage unbalance factor is defined in detail in (IEC 61000-4-30).

\section{Voltage fluctuations}

Voltage fluctuations can cause light intensity fluctuations that can be perceived by our brains. This effect, popularly known as flicker, can cause significant physiological discomfort. More precisely, flicker is the impression of unsteadiness of visual sensation induced by a light stimulus whose luminance or spectral distribution properly fluctuates with time. The most common reference for flicker measurement is (IEC 61000-4-15), and the minimum measurement period is one week.

\section{E. Voltage sags}

A single voltage dip can be characterized according to (IEC 61000-4-30) by a pair of data (residual voltage or depth and duration) or by a single index obtained by properly handling the aforementioned pair of data (the voltage sag aggressiveness index, voltage tolerance curvebased indices, the voltage dip energy index or the voltage sag severity index) (IEEE Std. 1564).

\section{F. Transient overvoltage}

A transient overvoltage can be classified as oscillatory or impulsive. An oscillatory transient event is described by its voltage peak, predominant frequency and decay time (duration). 
An impulsive transient event is described by rise time, decay time and peak value. The characterization of transient events is, in most cases, only based on peak value and duration. The peak value of transient overvoltage is the highest absolute value of the voltage waveform; the duration is the amount of time during which the voltage is above a threshold. The choice of the threshold level will affect the value of the duration. The higher the threshold level, the lower the resulting value for the duration. A suitable choice for the threshold used to calculate the duration would be the same as that used to detect the transient. Obviously, the conventional PQ indices such as the total harmonic distortion (THD), flicker factor, imbalance factor, etc., characterize specific types of power disturbances rather than their overall effects, and these indices are based on measurements made within an observation window, therefore they are not able to reveal the time varying characteristic of even a specific type of power disturbance. In additional, under practical circumstances, a PQ event usually consists of a combination of the power disturbances and most power disturbances are non-stationary. In consequence, the conventional PQ indices cannot completely satisfy practical applications. Therefore, desirable PQ indices need to characterize the overall effects of these power disturbances rather than a specific type and address the overall negative impact of the distorted waveform. At the same time, desirable $\mathrm{PQ}$ indices should be able to reflect the time varying characteristics of power disturbances as well as the distorted waveform caused by these disturbances.

\subsection{Novel power quality indices}

The transient disturbances occurring in power system can be considered as non-stationary and containing multiple frequency components. Therefore, new power quality indices should be able to reflect the time varying characteristics of transient disturbances as well as the distorted waveform caused by these disturbances. By not translating the oscillatory exponential kernel, S-transform localizes the real and the imaginary components of the spectrum independently, localizing the phase spectrum as well as the amplitude spectrum. Thus based on the good time-frequency localization properties of S-transform, four new power quality indices are introduced in this section to evaluate the transient disturbances.

\section{A. Instantaneous RMS (IRMS)}

The IRMS is defined as

$$
\mathrm{IRMS}=\sqrt{0.5 \times\left(\sum_{n}\left(A_{1}\left[k T, n f_{1}\right]\right)^{2}\right)}
$$

where $A_{1}$ is the amplitude spectrum calculated from the S-matrix of the disturbance signal. IRMS can be considered as a transient version of RMS accommodating the time information and contain all fundamental and harmonic components in the disturbance signal. As an instantaneous value, IRMS represents the RMS varying over time.

\section{B. Instantaneous Harmonic Distortion Ratio (IHDR)}

The IHDR is defined as

$$
\mathrm{IHDR}=\frac{\sqrt{\sum_{n \neq 1}\left(A_{d}\left[k T, n f_{1}\right]\right)^{2}}}{A_{0}\left[k T, f_{1}\right]} \times 100 \%
$$


where $A_{d}=A_{1}-A_{0}$ and $A_{1}, A_{0}$ are the amplitude spectrum of the disturbance signal and pure sinusoid signal respectively. Both the signals are normalized in unit before the S-transform processing. THD is a very common power quality index which estimates the overall deviation of distorted signal for its fundamental. The definition of the IHDR in the S domain is interpreted as the harmonic component in the disturbance signal divided by the fundamental component of the pure sinusoid signal. Thus IHDR represents the timevarying nature of the harmonic component relative to the sinusoid fundamental and correspondingly, does not subject to the impact of fundamental frequency RMS of the low frequency disturbances.

\section{Instantaneous Waveform Distortion Ratio (IWDR)}

The IWDR is defined as

$$
\mathrm{IWDR}=\sqrt{\frac{\sum_{n}\left(A_{d}\left[k T, n f_{1}\right]\right)^{2}}{\sum_{n}\left(A_{0}\left[k T, n f_{1}\right]\right)^{2}}} \times 100 \%
$$

where $A_{d}, A_{0}$ denote the same definition in (16). IWDR is a global index which represents the degree of disturbance waveform distortion from the pure sinusoid with unit amplitude. Different form the IHDR defined in equation (16), the molecule contains the fundamental component distortion of the transient disturbances. So IHDR can represent the waveform distortion caused by a low frequency disturbance such as interruption, sag or swell.

\section{Instantaneous Average Frequency (IAF)}

The IAF is defined as

$$
\mathrm{IAF}=\frac{\sum_{n}\left(n f_{1} \times A_{1}\left[k T, n f_{1}\right]\right)^{2}}{\sum_{n}\left(A_{1}\left[k T, n f_{1}\right]\right)^{2}}
$$

where $A_{1}$ denote the same definition in equation (16). Frequency is a very common physical quantity to describe the signal, but for the non-stationary signal with time-varying frequency, another frequency concept, instantaneous frequency should be introduced. Taking into account the disturbance signal containing the multiple frequency components, the IAF is calculated from the S-matrix using a first-order moment (Shin et al., 2006). Consequently, typical power system waveforms are characterized by a fundamental component that is the main component at a rated frequency and in the presence of a disturbance, larger frequency content will result in higher value of IAF than lower frequency content. In practice, IAF provides the deviation of the frequency from the fundamental frequency due to the presence of harmonic components.

\section{Application examples}

The performance of the four new power quality indices is evaluated using mathematical and PSCAD/EMTDC simulated disturbance signals. Firstly, in order to investigate the accuracy of S-transform based indices in power quality evaluation, transient disturbance signal 
represented mathematically with known parameters are tested. Then two PSCAD/EMTDC simulated disturbance signals due to ground fault and capacitor switching are analyzed using four power quality indices. The sampling frequency for all the transient disturbances is $5 \mathrm{kHz}$.

\subsection{Mathematical transient disturbances}

Case1 is low frequency disturbance as voltage sag, swell and interruption that can be expressed mathematically as

$$
s(t)=\left(1+\alpha\left(u\left(t_{2}\right)-u\left(t_{1}\right)\right)\right) \sin \left(2 \pi f_{0} t\right)
$$

where $f_{0}$ is $50 \mathrm{~Hz}, \mathrm{t}_{1}$ and $\mathrm{t}_{2}$ are the disturbance starting and ending time respectively, $\mathrm{a}$ is amplitude change factor: $a=-0.1 \sim-0.9$ corresponding to voltage sag, $a=0.1 \sim 0.8$ corresponding to swell and $-1 \leq a \leq-0.9$ corresponding to interruption.

There $30 \%$ voltage sag, $50 \%$ swell and interruption are constructed using (10) by set $a=-0.3$, $0.5,-0.9$ respectively. The disturbances start at $t_{1}=0.1 \mathrm{~s}$ and end at $t_{2}=0.28 \mathrm{~s}$. The low frequency disturbance waveforms and S-transform based time frequency distributions are shown is Fig.1. It is can seen that not only fundamental component vary during disturbances occur, but also high frequency component exist at disturbance start and end time.

Fig. 2 shows the four power quality indices of three low frequency disturbances. The IRMS of voltage sag, swell, and interruption in Fig.2(a) is 0.495, 1.061, and 0.0707 respectively during the disturbance occurred, but 0.707 at other time. In Fig.2(b), the IHDR shows two local maximum values corresponding to the start and end time of the three disturbances. The peak values of IHDR for voltage sag, swell, and interruption are $4.63 \%, 7.93 \%, 14.6 \%$ located exactly at $0.1 \mathrm{~s}$ and $3.77 \%, 6.6 \%, 12.2 \%$ at $0.28 \mathrm{~s}$. The IWDR of sag, swell and interruption in Fig.2(c) represents the distortion with the value 30\%, 50\%and 90\% from the pure sinusoid, which are consistent with the initial amplitude parameters respectively. With the same local maximum values similar to IHDR, the IAF in Fig.2(d) represents the timevarying instantaneous average frequency for the three disturbances and respective peak values are $51.8 \mathrm{~Hz}, 52.3 \mathrm{~Hz}, 85 \mathrm{~Hz}$ and $50.5 \mathrm{~Hz}, 50.7 \mathrm{~Hz}, 60 \mathrm{~Hz}$.

It can be concluded that these indices effectively represent the transient characteristics of low frequency disturbances. The value of sag amplitude is 0.7 and the corresponding RMS is 0.7 divided by 1.414 , which is equal to 0.495 . The right results are also represented for voltage swell and interruption; therefore, IRMS accurately represents the RMS varying over time. Compared Fig.2(c) with Fig.2(b), the greater IWDR corresponds with a larger IHDR, this is because there is a larger amplitude change at the start/end time. The IAF shows the similar quantitative relationship corresponding to IWDR and IAF is less deviation from 50 $\mathrm{Hz}$ as few high frequency components contained in low frequency disturbances, especially when the disturbances start or end at the zero-crossing point.

In Case2, transient oscillation signal which is a simulation of a capacitor switching event is expressed as

$$
s(t)=\sin \left(2 \pi f_{0} t\right)+\beta e^{-\left(t-t_{1}\right) / \tau} \sin \left(2 \pi f_{1} t\right)\left(u\left(t_{2}\right)-u\left(t_{1}\right)\right)
$$

where, $f_{0}$ and $f_{1}$ is the fundamental and transient frequency respectively, $t_{1}$ and $t_{2}$ are the oscillation starting and ending time, $\beta$ is the amplitude of exponential function, $\tau$ is the decay time coefficient. 


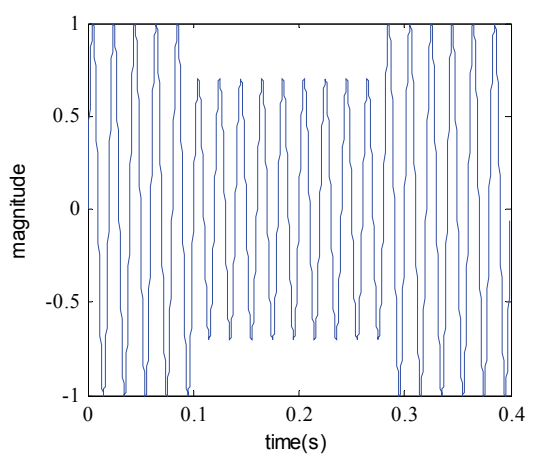

a)

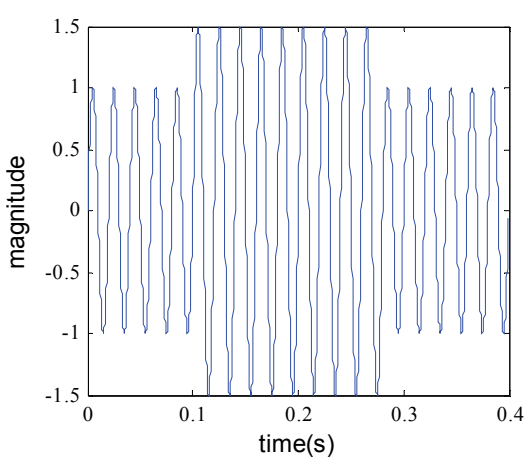

c)

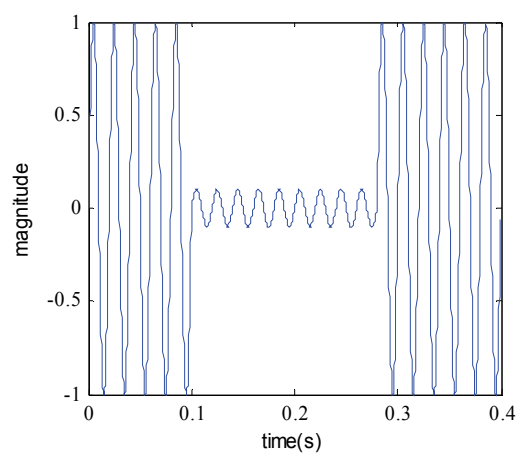

e)

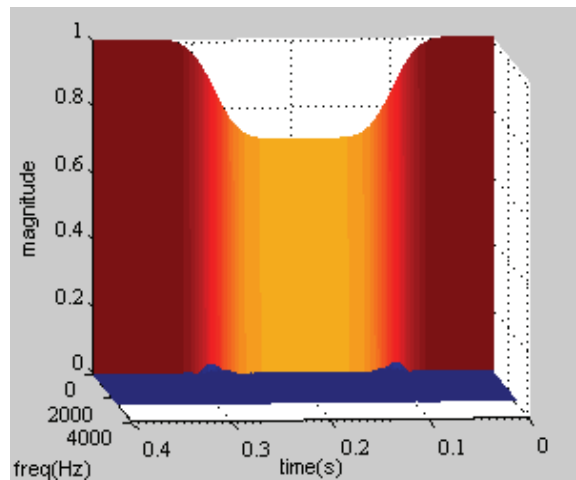

b)

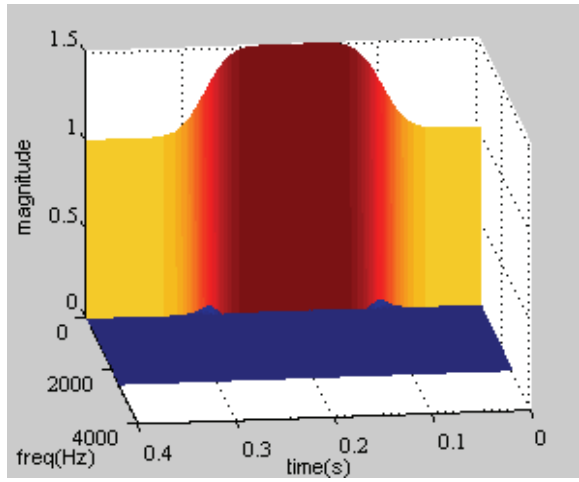

d)

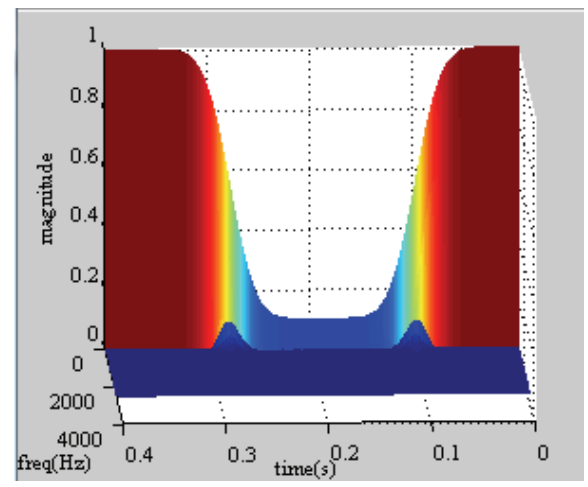

f)

Fig. 1. Low frequency disturbances and S-transient based time-frequency distributions: (a) Voltage sag waveform. (b) Time frequency distribution of voltage sag. (c) Voltage swell waveform. (d) Time frequency distribution of voltage swell. (e) Voltage interruption waveform. (d) Time frequency distribution of voltage interruption 


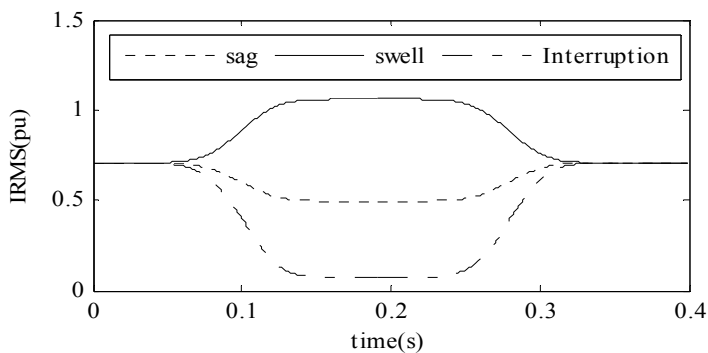

a)

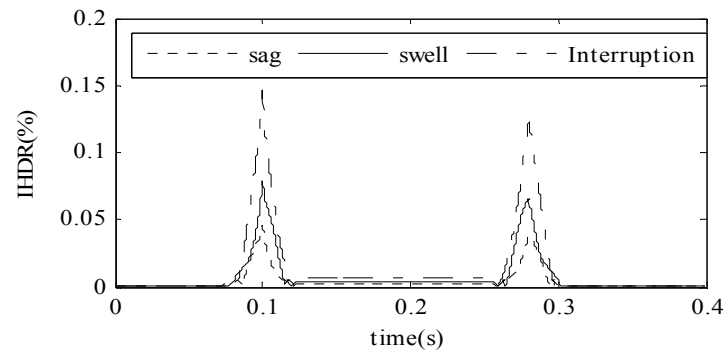

b)

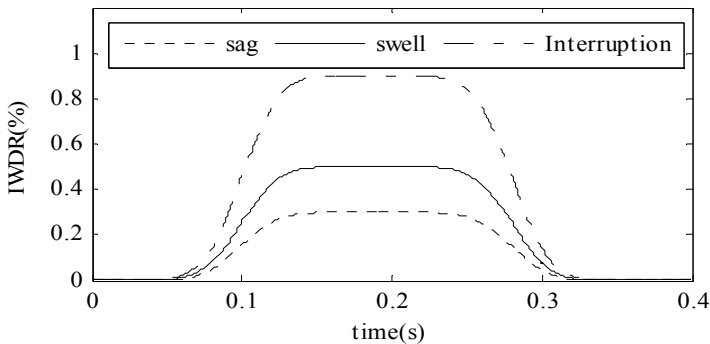

c)

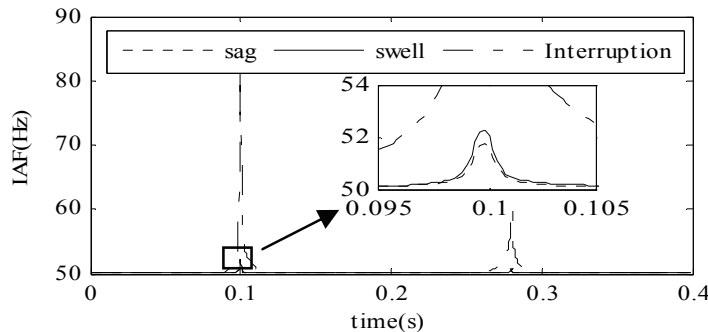

d)

Fig. 2. S-transform based power quality indices of voltage sag, swell and interruption: (a) Instantaneous RMS (IRMS). (b) Instantaneous Harmonic Distortion Ratio (IHDR) (c) Instantaneous Waveform Distortion Ratio (IWDR). (d) Instantaneous Average Frequency (IAF) 


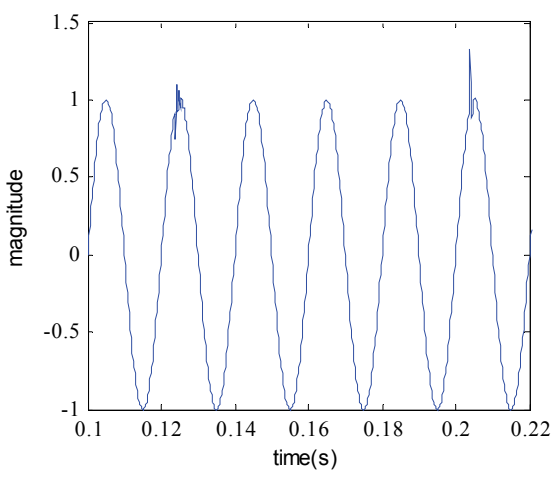

a)

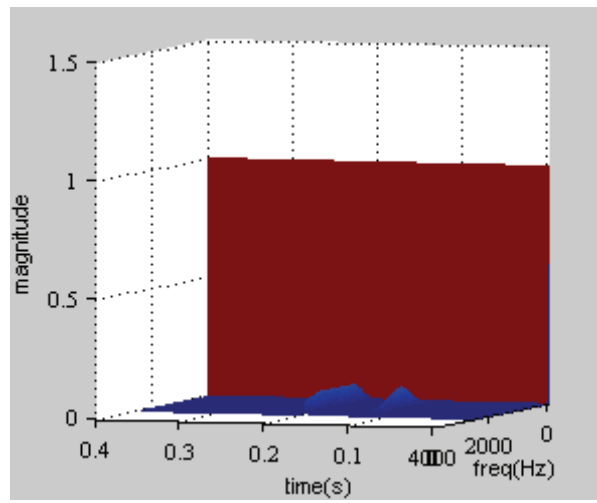

b)

Fig. 3. Transient oscillations and S-transient based time-frequency distribution: (a) Transient oscillation waveform. (b) Time frequency distribution of Transient oscillations

In Fig. 3(a), transient oscillation signal contains two oscillations superposed on the pure sinusoid. The first disturbance is fast oscillation started at $t=0.124 \mathrm{~s}\left(f_{1}\right.$ is $1500 \mathrm{~Hz}, \beta$ is 0.2 and $\tau$ is 2.5$)$ and the second disturbance is slow oscillation started at $t=0.204 \mathrm{~s}\left(f_{1}\right.$ is $600 \mathrm{~Hz}, \beta$ is 0.4 and $\tau$ is 5). The time-frequency distribution based on S-transform is shown in Fig. 3(b). The IRMS in Fig.4(a) shows two local maximum values 0.754 at $0.124 \mathrm{~s}$ and 0.795 at $0.204 \mathrm{~s}$ corresponding to the fast and slow oscillation respectively, which corresponds to the initial amplitude parameter $\beta=0.2$ and $\beta=0.4$. Moreover, the IRMS of the signal in steady-state time is 0.707 exactly. As there is almost no distortion in fundamental component of the transient oscillation compared with pure sinusoid, that is different from the low frequency disturbance in case 1, the IHDR in Fig.4(b) and the IWDR in Fig.4(c) represent a very similar result with peak value $37.1 \%$ and $50.8 \%$ at the time oscillations occurred. The IAF represents the deviation of average frequency from $\mathrm{f} 0$ and shows peak values $235 \mathrm{~Hz}$ and $339 \mathrm{~Hz}$ in Fig.4(d) for the fast and slow oscillation. Though the fast oscillation has a higher frequency component, the slow oscillation induces a greater IAF value than the fast oscillation due to its larger oscillation amplitude and accordingly greater spectral content of high frequency. 


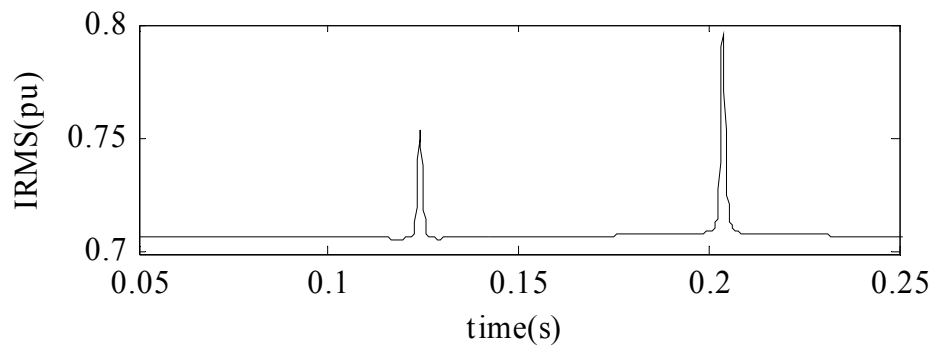

a)

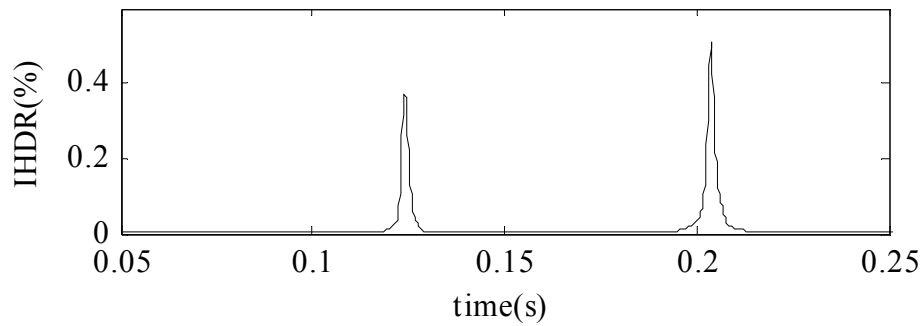

b)
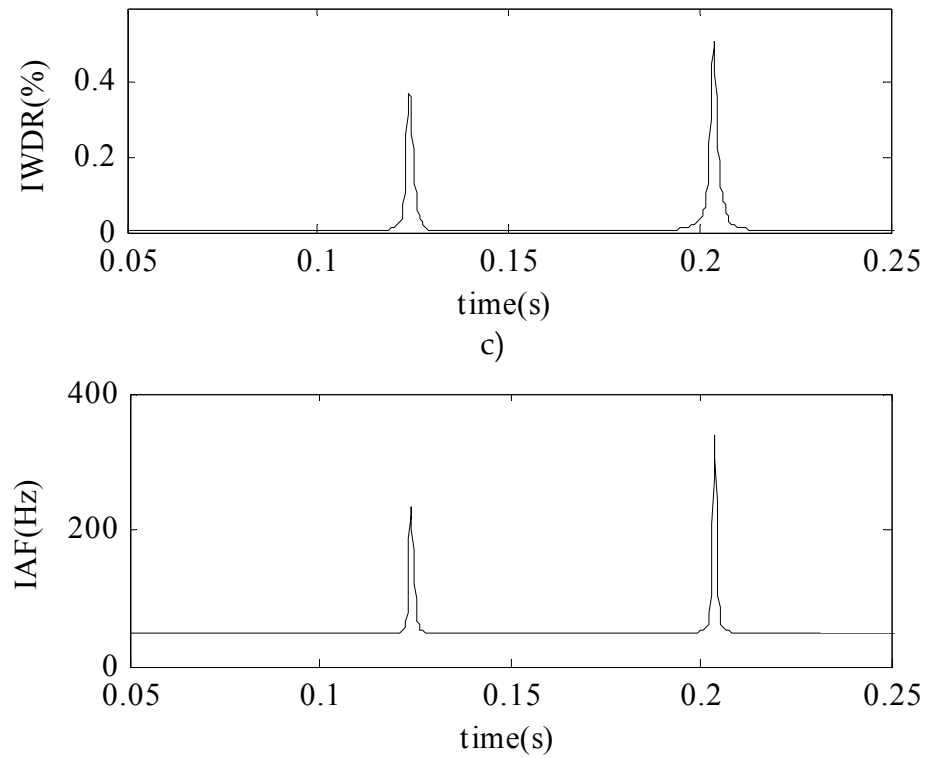

d)

Fig. 4. S-transform based power quality indices of transient oscillations: (a) Instantaneous RMS (IRMS). (b) Instantaneous Harmonic Distortion Ratio (IHDR) (c) Instantaneous Waveform Distortion Ratio (IWDR). (d) Instantaneous Average Frequency (IAF)

Concluded from the low frequency disturbances and transient oscillations with known parameters analyzed in the case 1 and case2, the results show that the four power quality 
indices accurately represent the transient characters of the transient disturbances. IRMS can accurately represent the RMS accommodating the time information. IHDR mainly represents the harmonic component relative to the pure sinusoid fundamental. However, IWDR focuses on the fundamental component distortion of the transient disturbances and also the harmonic distortion. Therefore there is the similar result between IHDR and IWDR when the transient oscillation is analyzed, that is very different from the results of low frequency disturbances. IAF represents the instantaneous average frequency of the transient disturbances and denotes the rated frequency when there is no disturbance occurred.

\subsection{PSCAD/EMTDC simulated disturbances}

A simple distribution model is built in PSCAD/EMTDC and two transient disturbances: voltage sag and capacitor switching which are two most common disturbances are obtained to illustrate the performance of four power quality indices.

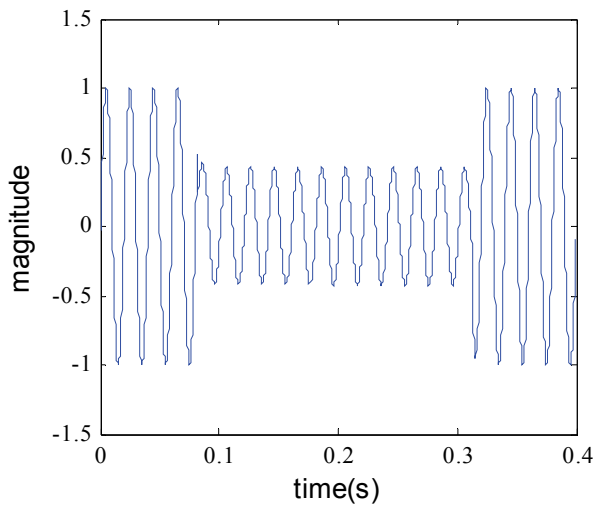

a)

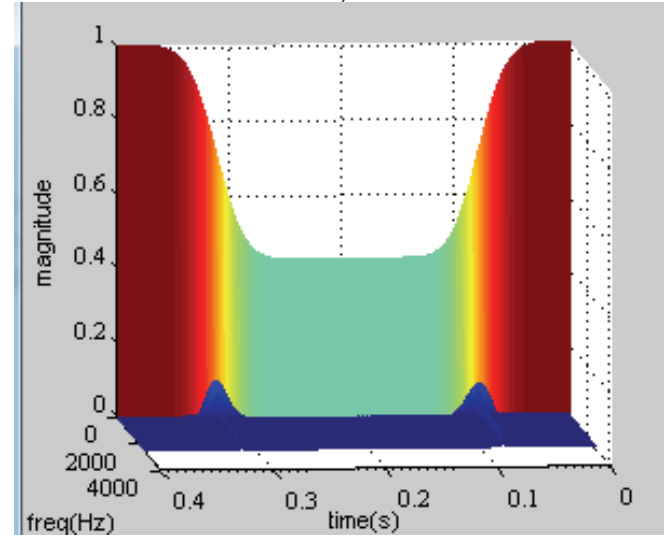

b)

Fig. 5. Voltage sag due to A phase grounded fault: (a) Voltage sag waveform. (b) Stransform based time frequency distribution of voltage sag 
A voltage sag caused by A phase grounded fault is simulated and the waveform of A phase voltage is shown in Fig. 5(a). Fig. 5(b) shows the time frequency distribution based on Stransform. The disturbance occurs at $0.082 \mathrm{~s}$ and ends at $0.313 \mathrm{~s}$. The four power quality indices: RMS, IHDR, IWDR and IAF are calculated and a summary of these indices is show in Tab. 1. Similar to the results of the voltage sag in case1, the IRMS is 0.3 and the IWDR is $57.6 \%$ during the disturbance occurred. The steady values of IRMS and IWDR are 0.707 and 0 . There are also two peaks in the IHDR and IAF corresponding to the start and end time, which are $28.2 \%$ and $190 \mathrm{~Hz}$ at $0.082 \mathrm{~s}$ and $22.3 \%$ and $109 \mathrm{~Hz}$ at $0.313 \mathrm{~s}$ respectively. Compared with the voltage sag in case1, this disturbance is not start/end at the zero-crossing point; moreover, there is a larger amplitude change with the IWDR value $57.6 \%$. Consequently, more harmonic content is contained in the disturbance signal, leading to a higher IHDR and a higher IAF.

\begin{tabular}{|c|c|c|c|}
\hline \multicolumn{2}{|c|}{ indices } & transient & steady \\
\hline \multicolumn{2}{|c|}{ IRMS $(\mathrm{pu})$} & 0.3 & 0.707 \\
\hline \multicolumn{2}{|c|}{ IWDR $(\%)$} & 57.6 & 0 \\
\hline \multicolumn{2}{|c|}{ indices } & start & end \\
\hline \multirow{2}{*}{ IHDR } & $\mathrm{t}(\mathrm{s})$ & 0.082 & 0.313 \\
\cline { 2 - 4 } & peak $(\%)$ & 28.2 & 22.3 \\
\hline \multirow{2}{*}{ IAF } & $\mathrm{t}(\mathrm{s})$ & 0.082 & 0.313 \\
\cline { 2 - 4 } & peak $(\mathrm{Hz})$ & 190 & 109 \\
\hline
\end{tabular}

Table 1. S-transform based four indices of voltage sag

Another disturbance as transient oscillation due to capacitor switching is showed in Fig.6 and the 0.3MVAR capacitor is put into operation at 0.153s. Tab.2. provides the transient peak values and steady values of the four indices. The peak value of IRMS is 0.722 at $0.153 \mathrm{~s}$ and the peak value of IWDR is $20.1 \%$ at the same time that is almost equivalent to the IHDR. The IAF also has a peak value $98 \mathrm{~Hz}$ when transient oscillation occurred and maintain at $50 \mathrm{~Hz}$ once the oscillation ended. As the IRMS is a little deviation from the rated value, there is less harmonic content in the disturbance. Accordingly, the value of IHDR, IWDR and IWDR is smaller relative to the disturbance in case2.

Obviously, the two transient disturbances as voltage sag and capacitor switching are characterized well by the four power quality indices. Therefore one can accurately represent the transient information over the time based on the good time-frequency localization properties of S-transform.

\begin{tabular}{|c|c|c|}
\hline indices & Transient (peak) & steady \\
\hline IRMS $(\mathrm{pu})$ & 0.722 & 0.707 \\
\hline IHDR $(\%)$ & 20.1 & 0 \\
\hline IWDR $(\%)$ & 20.1 & 0 \\
\hline IAF $(\mathrm{Hz})$ & 98 & 50 \\
\hline
\end{tabular}

Table 2. S-transform based four indices of capacitor switching 


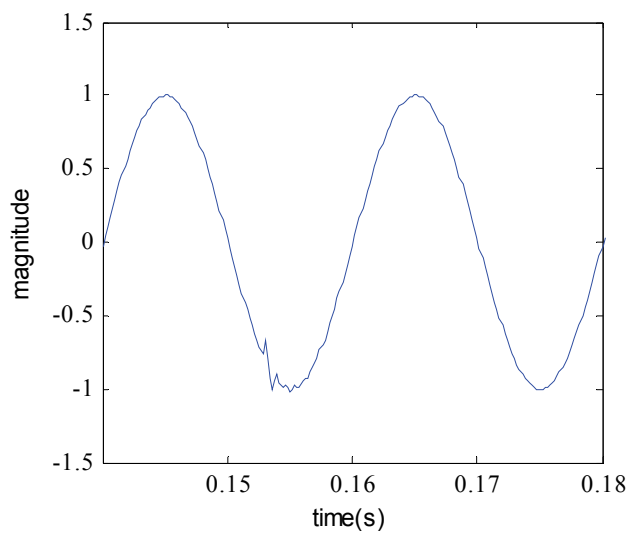

a)

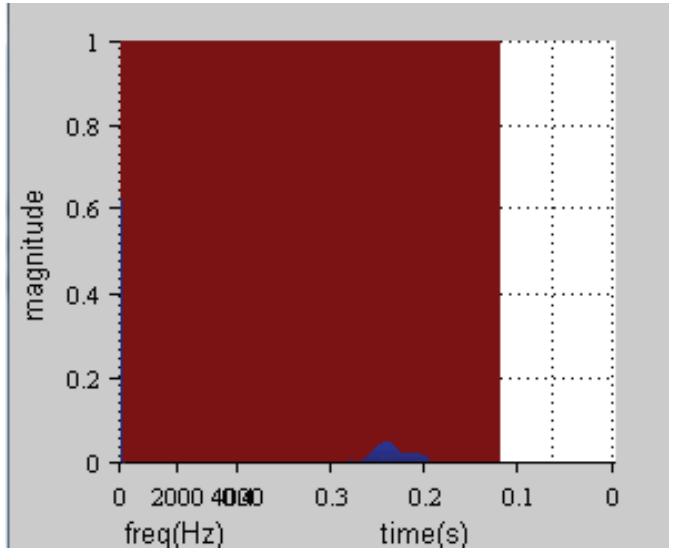

b)

Fig. 6. Transient oscillation due to capacitor switching: (a) capacitor switching waveform. (b) S-transform based time frequency distribution of transient oscillation

\section{Conclusion}

In this chapter, power quality assessment for transient disturbance signals has been carefully treated based on S-transform. The limitations of the traditional Fourier series coefficient based power quality indices, which inherently require periodicity of the disturbance signal, have been resolved by use of time-frequency analysis. In order to overcome the limitations of the traditional power quality indices in analyzing transient disturbances which are non-stationary waveforms with time-varying spectral component, four instantaneous power quality indices based on S-transform are presented. S-transform is shown to be a new time frequency analysis tool producing instantaneous time frequency representation with frequency dependent resolution. In the S-transform domain, new power quality indices: IRMS, IHDR, IWDR and IAF are defined and discussed. The effectiveness of these indices was tested using a set of disturbances represented mathematically and 
simulated in PSCAD/EMTDC respectively. The results show that the instantaneous property of transient disturbance can be characterized accurately.

The transient power-quality indices provide useful information about the time varying signature of the transient disturbance for assessment purposes. However, if the timevarying signature can be quantified as a single number, it would be more informative and convenient for an assessment and comparison of transient power quality. The power quality indices proposed in this chapter can be extended to general indices assessment, which should collapse to the standard definition for the periodic case and also be calculable by a standard algorithm that yields consistent results. It is a subject of future research.

\section{References}

Beaulieu, G.; Bollen, M. H. J.; Malgarotti, S. \& Ball, R.(2002). Power quality indices and objectives: Ongoing activates in CIGREWG36-07, Proc. 2002 IEEE Power Engineering Soc. Summer Meeting, pp. 789-794.

Bollen, M. and Yu Hua Gu, I. (2006). Signal Processing of Power Quality Disturbances, Wiley IEEE Press, New Jersey.

CENELEC EN 50160, Voltage characteristics of electricity supplied by public distribution systems.

Chilukuri, M.V. \& Dash, P.K.(2004). Multiresolution S-transform-based fuzzy recognition system for power quality events, IEEE Trans. Power Delivery, vol. 19, no. 1, pp.323330.

Domijan, A.; Hari, A. \& Lin, T. (2004). On the selection of appropriate wavelet filter bank for power quality monitoring, Int. J. Power Energy Syst., Vol. 24, pp.46-50.

Gallo, D., Langella, R. \& Testa, A. (2002). A Self Tuning Harmonics and Interharmonics Processing Technique, European Transactions on Electrical Power, 12(1), 25-31.

Gallo, D., Langella, R. \& Testa, A. (2004). On the Processing of Harmonics and Interharmonics: UsingHanning Windowin Standard Framework, IEEE Transactions on Power Delivery, 19(1), 28-34.

Gargoom, A.M., Ertugrul, N. and Soong, W.L. (2005) A comparative study on effective signal processing tools for power quality monitoring, The 11th European Conference on Power Electronics and Applications (EPE), pp.11-4 .

Heydt G. T. \& Jewell W. T.(1998). Pitfalls of electric power quality indices, IEEE Trans. Power Delivery, vol. 13, no. 2, pp. 570-578.

Heydt, G. T.(2000). Problematic power quality indices, IEEE Power Eng. Soc. Winter Meeting, vol. 4, pp. 2838-2842.

IEEE Recommended Practice for Monitoring Electric Power Quality. (1995). IEEE Std. 11591995.

IEC 61000-3-6, Assessment of emission limits for distorting loads in MV and HV power systems.

IEC 61000-4-7, General guide on harmonics and interharmonics measurements and instrumentation for power supply systems and equipment connected thereto.

IEC 61000-4-15, Flickermeter, functional design and specifications.

IEC 61000-4-30, Power quality measurement methods. 
Jaramillo, S.H.; Heydt, G.T. \& O’Neill-Carrillo, E. (2000) ‘Power quality indices for a periodic voltages and currents', IEEE Transactions on Power Delivery, April, Vol. 15, No. 2, pp.784-790.

Lin, T. \& Domijan, A.(2005). On power quality Indices and real time measurement, IEEE Trans. Power Delivery, vol. 20, no. 4, pp.2552-2562.

Mishra, S.; Bhende, C.N. \& Panigrahi. B.K. (2008) Detection and classification of power quality disturbances using S-transform and probabilistic neural network, IEEE Trans. Power Delivery, vol. 23, no. 1, pp. 280-287.

Morsi, W. G. \& EI-Hawary, M. E. (2008). A new perspective for the IEEE standard 1459-2000 via stationary wavelet transform in the presence of non-stationary power quality disturbance, IEEE Trans. Power Delivery, vol. 23, no. 4, pp. 2356-2365.

Shin, Y. J.; Powers, E. J.; Grady, M. \& Arapostathis, A.(2006) Power quality indices for transient disturbances, IEEE Trans. on Power Delivery, vol. 21, no. 1, pp.253-261.

Stockwell, R. G.; Mansinha, L. \& R. P. Lowe (1996). Localization of the complex spectrum: The S-transform, IEEE Trans. Signal Processing, vol.144, pp. 998-1001.

Ward, D.J. (2001). Power quality and the security of electricity supply, Proceedings of the IEEE, pp.1830-1836.

Voltage sag indices draft 2, working document for IEEE P1564, December 2001.

Zhan, Y.; Cheng, H. Z. \& Ding, Y. F.(2005) S-transform-based classification of power quality disturbance signals by support vector machines, Proceedings of the CSEE, vol. 25, no. 4, pp. 51-56. 


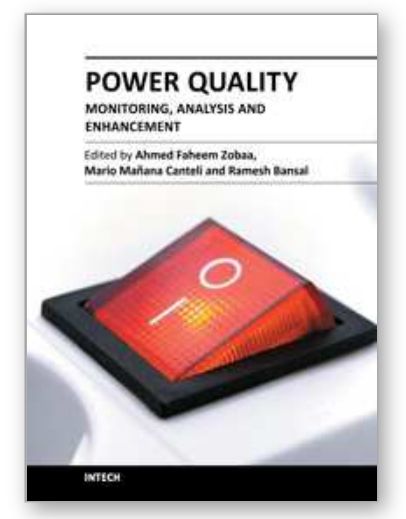

\author{
Power Quality â€“Â Monitoring, Analysis and Enhancement \\ Edited by Dr. Ahmed Zobaa
}

ISBN 978-953-307-330-9

Hard cover, 364 pages

Publisher InTech

Published online 22, September, 2011

Published in print edition September, 2011

This book on power quality written by experts from industries and academics from various counties will be of great benefit to professionals, engineers and researchers. This book covers various aspects of power quality monitoring, analysis and power quality enhancement in transmission and distribution systems. Some of the key features of books are as follows: Wavelet and PCA to Power Quality Disturbance Classification applying a RBF Network; Power Quality Monitoring in a System with Distributed and Renewable Energy Sources; Signal Processing Application of Power Quality Monitoring; Pre-processing Tools and Intelligent Techniques for Power Quality Analysis; Single-Point Methods for Location of Distortion, Unbalance, Voltage Fluctuation and Dips Sources in a Power System; S-transform Based Novel Indices for Power Quality Disturbances; Load Balancing in a Three-Phase Network by Reactive Power Compensation; Compensation of Reactive Power and Sag Voltage using Superconducting Magnetic Energy Storage; Optimal Location and Control of Flexible Three Phase Shunt FACTS to Enhance Power Quality in Unbalanced Electrical Network; Performance of Modification of a Three Phase Dynamic Voltage Restorer (DVR) for Voltage Quality Improvement in Distribution System; Voltage Sag Mitigation by Network Reconfiguration; Intelligent Techniques for Power Quality Enhancement in Distribution Systems.

\title{
How to reference
}

In order to correctly reference this scholarly work, feel free to copy and paste the following:

Zhengyou He and Yong Jia (2011). S-Transform Based Novel Indices for Power Quality Disturbances, Power Quality âE"Â Monitoring, Analysis and Enhancement, Dr. Ahmed Zobaa (Ed.), ISBN: 978-953-307-330-9, InTech, Available from: http://www.intechopen.com/books/power-quality-monitoring-analysis-andenhancement/s-transform-based-novel-indices-for-power-quality-disturbances

\section{INTECH}

open science | open minds

\author{
InTech Europe \\ University Campus STeP Ri \\ Slavka Krautzeka 83/A \\ 51000 Rijeka, Croatia \\ Phone: +385 (51) 770447 \\ Fax: +385 (51) 686166 \\ www.intechopen.com
}

\author{
InTech China \\ Unit 405, Office Block, Hotel Equatorial Shanghai \\ No.65, Yan An Road (West), Shanghai, 200040, China \\ 中国上海市延安西路65号上海国际贵都大饭店办公楼405单元 \\ Phone: +86-21-62489820 \\ Fax: $+86-21-62489821$
}


(C) 2011 The Author(s). Licensee IntechOpen. This chapter is distributed under the terms of the Creative Commons Attribution-NonCommercialShareAlike-3.0 License, which permits use, distribution and reproduction for non-commercial purposes, provided the original is properly cited and derivative works building on this content are distributed under the same license. 